\title{
Cognitive Frailty and 30-Day Mortality in a National Cohort of Older Chinese Inpatients
}

This article was published in the following Dove Press journal: Clinical Interventions in Aging

\author{
Xiao-Ming Zhang ${ }^{\text {,* }}$ \\ Jing Jiao ${ }^{1, *}$ \\ Chen Zhu' \\ $\mathrm{Na}$ Guo' \\ Ying Liu' \\ Dongmei $\mathrm{Lv}^{2}$ \\ Hui Wang ${ }^{3}$ \\ Jingfen Jin ${ }^{4}$ \\ Xianxiu Wen ${ }^{5}$ \\ Shengxiu Zhao ${ }^{6}$ \\ Xin-Juan $\mathrm{Wu}^{1}$ \\ Tao $\mathrm{Xu}^{7}$
}

'Department of Nursing, Chinese Academy of Medical Sciences - Peking Union Medical College, Peking Union Medical College Hospital (Dongdan Campus), Beijing, 100730, People's Republic of China; ${ }^{2}$ Department of Nursing, The Second Affiliated Hospital of Harbin Medical University, Harbin, People's Republic of China; ${ }^{3}$ Department of Nursing, Tongji Hospital, Tongji Medical College, Huazhong University of Science and Technology, Wuhan, People's Republic of China; ${ }^{4}$ Department of Nursing, The Second Affiliated Hospital Zhejiang University School of Medicine, Hangzhou, People's Republic of China; ${ }^{5}$ Department of Nursing, Sichuan Provincial People's Hospital, Chengdu, People's Republic of China; ${ }^{6}$ Department of Nursing, Qinghai Provincial People's Hospital, Xining, People's Republic of China; ${ }^{7}$ Department of Epidemiology and Statistics, Institute of Basic Medical Sciences, Chinese Academy of Medical Sciences \& School of Basic Medicine, Peking Union Medical College, Beijing, I00005, People's Republic of China

*These authors contributed equally to this work

Correspondence: Xin-Juan Wu; Tao Xu $\mathrm{Tel} / \mathrm{Fax}+01086$ 69I5 6II4 Email wuxinjuan@sina.com; xutaosd@I26.com
Purpose: Studies exploring the association of cognitive frailty and mortality have been mainly based on community settings or nursing home settings. The aim of our study was to explore the association between cognitive frailty and 30-day mortality among older Chinese inpatients.

Patients and Methods: A national cohort study was performed in different hospitals in China. A baseline survey was conducted from October 2018 and February 2019. Trained investigators collected the 30-day mortality. Cognitive impairment and frailty were defined by the Mini-Cog and FRAIL scale, respectively. Multivariate regression was used to explore the association between cognitive impairment and frailty status with 30-day mortality.

Results: Of these participants, there were 3891 (41.91\%) women and 5392 (58.09\%) men, with an average age of $72.41(\mathrm{SD}=5.72)$. The prevalence of cognitive frailty was $5.44 \%$. After adjusting for age, gender, education, depression and activities of daily living (ADL), the odds ratios (ORs) for 30-day mortality among inpatients were 3.43 (95\% CI: 1.80-6.55) for cognitive frailty, 1.85 (95\% CI: 1.01-3.41) for frailty only, and 1.43 (95\% CI: 0.77-2.65) for cognitive impairment only compared to the reference group (neither frailty nor cognitive impairment). In addition, the discrimination of 30-day mortality was higher among patients with cognitive frailty (area under the curve $=0.676$ [95\% CI: $0.621-0.731]$ ) than either frailty (area under the curve $=0.644$ [95\% CI: 0.594-0.694]) or cognitive impairment (area under the curve $=0.606$ [95\% CI: $0.556-0.655]$ ) separately. Stratified analysis showed that these associations still existed when grouped by gender.

Conclusion: Our study found that Chinese inpatients with cognitive frailty had a higher risk of 30-day mortality than those without frailty and cognitive impairment, suggesting that clinicians should be encouraged to perform early screening of patients with frailty and cognitive impairment and carry out effective interventions to reverse cognitive frailty syndrome.

Keywords: frailty, cognitive impairment, mortality, older adults, Chinese; inpatients

\section{Introduction}

Population aging is a worldwide demographic issue, corresponding to the increased longevity in older adults. These older adults often suffer from age-related comorbidities, with a higher likelihood of developing frailty and cognitive impairment when coexisting with stressors such as illness, injury and psychosocial stress. ${ }^{1}$ Frailty and cognitive impairment are prevalent among older adults. ${ }^{2,3}$ Both of these geriatric syndromes are reported in conjunction with an increased risk of mortality and other adverse outcomes, such as hospitalization and disability. ${ }^{4,5}$ Therefore, managing and identifying frailty and cognitive impairment is essential for improving health in this population. 
Currently, a great number of studies have found a close association between frailty and cognitive impairment, indicating that frailty and cognitive impairment are risk factors for one another. ${ }^{6,7}$ Frailty and cognitive impairment seem to have similar pathogenic mechanisms, with chronic inflammation, oxidative stress, and hormonal dysregulation likely playing an important role. ${ }^{8}$ In vulnerable older adults, these syndromes often cooccur, with the term "cognitive frailty" recommended by an international consensus group to define an older adult with the characteristics of both frailty and cognitive impairment but an absence of dementia. ${ }^{9}$ However, there is no consensus definition of cognitive frailty, and there is also no consensus assessment tool to measure frailty and cognitive function. Some studies have used the Fried frailty phenotype,${ }^{10}$ FRAIL scale, ${ }^{8}$ and Clinical Frailty Scale (CFS) ${ }^{11}$ to define frailty. There are also various assessment scales to measure cognitive impairment, such as the Mini-Mental State Examination (MMSE) ${ }^{12}$ and Montreal Cognitive Assessment (MoCA), which may result in different prevalence of cognitive frailty.

Cognitive frailty has been widely reported to be associated with mortality. Several studies have indicated that older adults suffering from cognitive frailty have the highest risk of mortality compared with those having separate frailty or cognitive impairment or being nonfrail with intact cognitive function. ${ }^{11,13-21}$ However, most of the abovementioned studies focused on community-dwelling adults $^{15,17}$ or nursing home residents. ${ }^{22}$ No study has reported this association among hospitalized older Chinese adults. Given that hospitalized older adults suffer worse health, it is therefore essential to examine the association between cognitive frailty and mortality among these vulnerable older patients in the hospital. Our objectives were to investigate the prevalence of cognitive frailty and to explore the association between cognitive frailty and 30-day mortality among hospitalized Chinese patients. We believe these findings could add new advances to this field.

\section{Patients and Methods \\ Study Design and Population}

As other published studies have reported, this study was a national large-scale longitudinal study of frailty among hospitalized older patients at tertiary hospitals ${ }^{23}$ in different centers in China. Detailed information on the sampling methods was described in a previous study. ${ }^{23}$ Briefly, researchers adopted two stages of cluster sampling methods to recruit participants from the selected hospital. The baseline survey was performed between October 2018 and February 2019. We collected the data, including demographic, medical, physiological and psychological conditions, via questionnaire interviews and assessments. This study was approved by the Review Board, and all patients signed the informed consent form (S-K540). In addition, protocol number ChiCTR1800017682 was noted in our published study. ${ }^{23}$ We included hospitalized participants aged $\geq 65$ years old, excluding patients that were unconscious, could not communicate with the trained investigators.

\section{Frailty Assessment}

The FRAIL scale was employed by investigators to assess frailty syndrome. The FRAIL scale consists of five simple questions to evaluate five patient domains, including fatigue, ambulation, resistance, weight loss and illness. ${ }^{24}$ The questions were as follows: (1) How much of the time did you feel tired during the past four weeks? (all of the time or most of the time was scored as 1 , others as 0 ); (2) Can you walk a couple of blocks by yourself without any aid and without any difficulty? (Yes=0, $\mathrm{No}=1$ ); (3) Can you walk up 10 steps without having to stop and rest, by yourself, without aid? (Yes=0, No=1); (4) How many illnesses did the doctor tell you that you have? (more than five was recorded as 1 , whereas when the number ranged from 0 to 4, it was recorded as 0 ). Detailed information on the illnesses was reported in a previous study. (5) Did you lose weight, at $5 \%$ or more, within the past six months? $\left(Y e s=1, \mathrm{No}_{0}=0\right.$ ). The frailty categories were classified as frailty ( $\geq 3$ points) or nonfrail ( $0-2$ points).

\section{Cognitive Impairment Assessment}

We used a Chinese version of Mini-Cog, with two components that included a three-item recalled words test and one simple scored clock, to assess cognitive function. ${ }^{25}$ The total points ranged from $0-5$, with a score of less than 3 points indicating cognitive impairment. This assessment was a simple, non-time-consuming screening tool and is widely employed in clinics and hospital wards.

Cognitive frailty was defined when patients met these two geriatric syndromes (frailty and cognitive impairment).

\section{Other Variables}

We also collected other important variables, including sociodemographic (age, gender, education, marital status, 
ethnicity), functional covariates (vision, hearing, urinary function), depression, nutritional status and length of hospital stay. Lifestyle variables, which were self-reported, included smoking status and alcohol consumption. Functional covariates were detected based on whether they influenced daily life (dysfunction and normal). Depression symptoms were assessed using the Geriatric Depression Scale (GDS) with the definition score $\geq 5$ points. ${ }^{26}$ Nutritional status was assessed by the Mini-Nutritional Assessment-Short Form (normal nutritional status: 12-14; at risk of malnutrition: 8-11; malnourished: $0-7){ }^{27}$ The activities of daily living (ADLs) were assessed using the Barthel index. ${ }^{28}$

\section{Follow-Up for Outcome}

After performing a baseline survey, trained investigators attended a standard training program to obtain the necessary skills to conduct telephone interviews for follow-up. Thirty-day mortality was identified and recorded by the trained investigators.

\section{Statistical Analysis}

We performed all statistical analyses using the SAS package, version 9.4, (SAS Institute Inc., Cary, NC, USA), with significant differences identified by a twosided $P$-value $<0.05$. We divided the cohort into the following four groups: cognitive frailty, having only frailty with intact cognitive function, nonfrail with cognitive impairment, and nonfrail with normal cognitive function. All variables are displayed as mean \pm standard deviation or proportions/frequency when the data are numerical variables and categorical variables, respectively. Student's $t$-test, chi square test and one-way ANOVA were conducted to detect the differences between various groups. Multivariate logistic regression analysis was used to identify the odds ratio (OR), as well as the pulsing confidence interval (CI) of association between the aforementioned cognitive frailty groups and 30-day mortality. Based on a previous study, we assume that patients with frailty and cognitive impairment have a higher risk of 30-day mortality than those in the other three groups. Additionally, we listed the raw model and three other adjusted models. Model 1 adjusted for age, gender, and education; Model 2 adjusted for the factors in Model 1 plus depression; Model 3 adjusted for ADL in addition to the factors from Model 2. We also performed subgroup analysis based on gender. Finally, receiver operating characteristic analysis was used to compare the area under the curve (AUC) among cognitive frailty, frailty alone, and cognitive impairment alone for predicting 30-day mortality.

\section{Results}

\section{Demographics}

The study flowchart is shown in Figure 1. We conducted a baseline survey that recruited 9996 patients from six provinces, resulting in 9283 participants after deleting incomplete data and participants lost to follow-up at 30 days. Overall, the average age was $72.41(\mathrm{SD}=5.72)$, with $41.91 \%$ female. The prevalence of frailty, cognitive impairment and depression was $16.91 \%, 20.51 \%$, and $16.17 \%$, respectively. Females had a significantly higher proportion of illiterate individuals than men $(23.65 \%$ versus $9.72 \%)$ and had a higher prevalence of frailty, cognitive impairment, and depression than males $(18.79 \%$ versus $15.56 \%$; $25.49 \%$ versus $16.91 \%$; 19.09 versus $14.07 \%$ ), as shown in Table 1

\section{Comparisons of Variables According to Frailty and Cognitive Impairment Status}

Overall, the prevalence of cognitive frailty was $5.44 \%$. Participants with cognitive frailty were older and more often female. Additionally, a significantly higher percentage were illiterate, suffered from depression, had lower handgrip strength and were malnourished (all $P<0.05$ ) compared with the other three groups. Furthermore, patients with cognitive frailty scored significantly lower in ADL and BMI (Table 2).

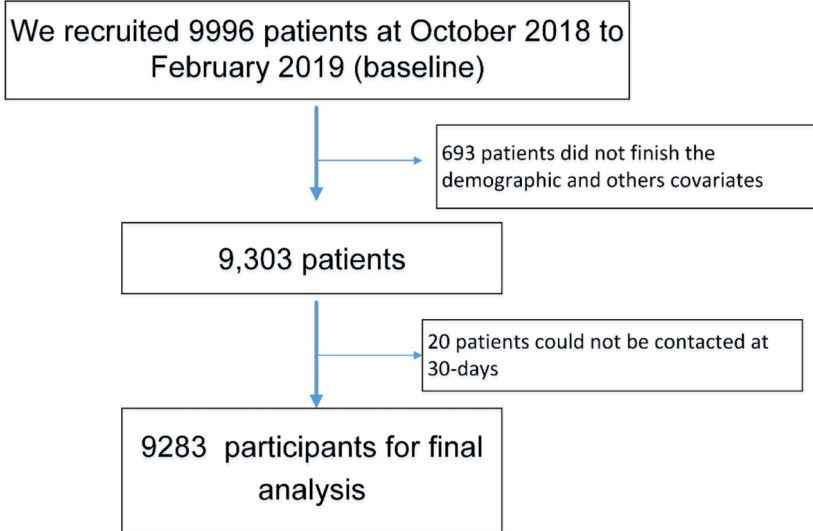

Figure I Flowchart of study selection. 
Table I Characteristics of the Study Population According to Gender

\begin{tabular}{|c|c|c|c|c|}
\hline Variables & Overall & Female & Male & P-value \\
\hline Sample & 9283 & $3891(41.91)$ & $5392(58.09)$ & \\
\hline Age (years) (mean, SD) & $72.4 I \pm 5.72$ & $72.28 \pm 5.50$ & $72.5 I \pm 5.87$ & 0.060 \\
\hline Education (n, \%) & & & & $<0.001$ \\
\hline Illiterate & I444(I5.56) & $920(23.65)$ & $524(9.72)$ & \\
\hline Primary & $2666(28.72)$ & $1136(29.20)$ & $1530(28.38)$ & \\
\hline Meddle & $3787(40.80)$ & $1417(36.43)$ & $2370(43.96)$ & \\
\hline University & $1384(|4.9|)$ & $417(10.72)$ & $967(17.94)$ & \\
\hline ADL (mean, SD) & $27.88 \pm 4.23$ & $27.65 \pm 4.37$ & $28.04 \pm 4.12$ & $<0.0001$ \\
\hline BMI $\left(\mathrm{kg} / \mathrm{m}^{2}\right)$ (mean, SD) & $23.61 \pm 3.49$ & $23.65 \pm 3.70$ & $23.57 \pm 3.34$ & 0.259 \\
\hline Ethnicity (n, \%) & & & & 0.299 \\
\hline Other & $510(5.49)$ & $225(5.78)$ & $285(5.29)$ & \\
\hline Han & $8773(94.5 I)$ & $3666(94.22)$ & $5107(94.7 I)$ & \\
\hline Marital status (n, \%) & & & & $<0.001$ \\
\hline Marriage & $8253(89.1)$ & $3206(82.52)$ & $5047(93.69)$ & \\
\hline Divorced or widowed & $1019(10.9)$ & $679(17.48)$ & $340(6.31)$ & \\
\hline Smoking status (n, \%) & & & & $<0.001$ \\
\hline Never smoking & $6104(65.75)$ & $3649(93.78)$ & $2455(45.53)$ & \\
\hline Current smoking & $1042(11.23)$ & $108(2.78)$ & $934(17.32)$ & \\
\hline Smoking before & $2137(23.02)$ & $134(3.44)$ & $2003(37.15)$ & \\
\hline Alcohol consumption (n, \%) & & & & $<0.001$ \\
\hline Never & $707 \mid(76.17)$ & $377 I(96.92)$ & $3300(61.20)$ & \\
\hline Current & $1096(11.81)$ & $78(2.00)$ & $1018(18.88)$ & \\
\hline Quit & $1116(12.02)$ & $42(1.08)$ & $1074(19.92)$ & \\
\hline Long-time bedridden (n, \%) & & & & 0.203 \\
\hline No & $9048(97.4)$ & $3783(97.22)$ & $5265(97.64)$ & \\
\hline Yes & $235(2.6)$ & $108(2.78)$ & $127(2.36)$ & \\
\hline Vision (n, \%) & & & & 0.0002 \\
\hline Yes & $1911(20.6)$ & $873(22.44)$ & $1038(19.25)$ & \\
\hline No & 7372(79.4) & $3018(77.56)$ & $4354(80.75)$ & \\
\hline Hearing (n, \%) & & & & 0.317 \\
\hline Yes & $166 \mid(\mid 7.89)$ & $678(17.42)$ & $983(18.23)$ & \\
\hline No & $7622(82.11)$ & $3213(82.58)$ & $4409(81.77)$ & \\
\hline Urinary function $(\mathrm{n}, \%)$ & & & & $<0.001$ \\
\hline
\end{tabular}


Table I (Continued).

\begin{tabular}{|c|c|c|c|c|}
\hline Variables & Overall & Female & Male & P-value \\
\hline Dysfunction & $1288(13.87)$ & $347(8.92)$ & $94 I(I 7.45)$ & \\
\hline Normal & $7995(86.12)$ & $3544(91.08)$ & $445 \mid(82.55)$ & \\
\hline Frailty $(\mathrm{n}, \%)$ & & & & $<0.001$ \\
\hline Yes & $1570(16.91)$ & $73 I(18.79)$ & $839(15.56)$ & \\
\hline No & $7713(83.08)$ & $3160(81.21)$ & $4553(84.44)$ & \\
\hline Cognitive impairment $(\mathrm{n}, \%)$ & & & & $<0.001$ \\
\hline No & 7379(79.48) & $2899(74.5 I)$ & $4480(83.09)$ & \\
\hline Yes & $1904(20.5 \mid)$ & $992(25.49)$ & $912(16.91)$ & \\
\hline Depression (n, \%) & & & & $<0.0001$ \\
\hline No & $7707(83.83)$ & $3116(80.91)$ & $4591(85.93)$ & \\
\hline Yes & $1487(16.17)$ & $735(19.09)$ & $752(14.07)$ & \\
\hline MNA-SF (n, \%) & & & & 0.136 \\
\hline $12-14$ & $508 I(54.73)$ & $2083(53.53)$ & $2998(55.60)$ & \\
\hline $8-11$ & $3224(34.73)$ & |39|(35.75) & $1833(34.00)$ & \\
\hline $0-7$ & $978(10.54)$ & $417(10.72)$ & $56 I(10.40)$ & \\
\hline Low Hand grip strength (n, \%) & & & & 0.034 \\
\hline Yes & $4742(51.08)$ & $2038(52.38)$ & $2704(50.15)$ & \\
\hline No & $454 I(48.92)$ & $1853(47.62)$ & $2688(49.85)$ & \\
\hline The length of hospital stay (mean, SD) & $9.67 \pm 7.23$ & $9.45 \pm 6.81$ & $9.82 \pm 7.51$ & 0.016 \\
\hline
\end{tabular}

Abbreviations: MNA-SF, Mini-Nutritional Assessment-Short Form (normal nutritional status:12-14; at risk of malnutrition: 8-II; malnourished:0-7).

\section{Univariate Analysis for the Variables Associated with 30-Day Mortality}

Overall, the average ADL and age in the deceased group was significantly higher than that of the surviving group (28.04 \pm 4.12 versus $27.65 \pm 4.37, P<0.0001 ; 72.4 \pm 5.7$ versus $73.8 \pm 6.1, P=0.014)$. In addition, the percentage of patients with cognitive frailty, depression and low handgrip strength in the deceased group was higher than that of the surviving group (all $P<0.001$ ), as shown in Table 3.

\section{Association Between Cognitive Frailty Status and 30-Day Mortality}

Table 4 indicates the association between 30-day mortality and cognitive frailty status. Overall, the ORs with
95\% CIs for 30-day mortality among patients with cognitive frailty, frailty alone, and cognitive impairment alone, compared to patients in the nonfrail and intact cognitive function group, were 6.83 (95\% CI: 3.91-11.95), 2.88 (95\% CI: 1.63-5.06), and 1.80 (95\% CI: $0.99-3.28)$, respectively in the raw model. Additionally, these associations for 30-day mortality in each category decreased after adjusting for different variables in different models. After adjusting for age, gender, education, depression and ADL, the ORs with 95\% CIs for 30-day mortality among patients with cognitive frailty, frailty alone, and cognitive impairment alone, compared to those who were nonfrail and with intact cognitive function, were 3.43 (95\% CI: 1.80-6.55), 1.85 (95\% CI: $1.01-3.41)$, and $1.43 \quad(95 \%$ CI: $0.77-2.65)$, respectively. 
Table 2 Characteristics of the Study Population According to Frailty and Cognitive Impairment Status

\begin{tabular}{|c|c|c|c|c|c|}
\hline Variables & $\begin{array}{l}\text { Frailty and } \\
\text { Cognitive } \\
\text { Impairment } \\
(505)\end{array}$ & $\begin{array}{l}\text { Frailty Only } \\
(1065)\end{array}$ & $\begin{array}{l}\text { Cognitive } \\
\text { Impairment } \\
\text { Only (I399) }\end{array}$ & $\begin{array}{l}\text { Nonfrail and with } \\
\text { Normal Cognitive } \\
\text { Function (6314) }\end{array}$ & P-value \\
\hline Age (years) (mean, SD) & $75.10 \pm 6.66$ & $73.23 \pm 5.99$ & $73.25 \pm 6.05$ & $71.87 \pm 5.41$ & $<0.0001$ \\
\hline ADL (mean, SD) & $23.4 I \pm 6.5 I$ & $25.2 I \pm 5.34$ & $27.96 \pm 4.16$ & $28.67 \pm 3.30$ & $<0.0001$ \\
\hline BMI $\left(\mathrm{kg} / \mathrm{m}^{2}\right)$ (mean, SD) & $22.61 \pm 3.76$ & $23.11 \pm 3.94$ & $23.44 \pm 3.69$ & $23.80 \pm 3.33$ & $<0.0001$ \\
\hline Gender (n, \%) & & & & & $<0.0001$ \\
\hline Female & $267(52.87)$ & $464(43.56)$ & $725(51.82)$ & $2435(38.56)$ & \\
\hline Male & $238(47.13)$ & $60 I(56.44)$ & $674(48.18)$ & $3879(61.44)$ & \\
\hline Ethnicity (n, \%) & & & & & $<0.0001$ \\
\hline Han & $433(85.74)$ & $987(92.67)$ & $1290(92.20)$ & 6063(96.02) & \\
\hline Other & $72(14.25)$ & $78(7.33)$ & $109(7.80)$ & $25 I(3.98)$ & \\
\hline Education (n, \%) & & & & & $<0.0001$ \\
\hline Illiterate & $193(38.21)$ & $159(14.93)$ & $408(29.16)$ & $684(10.83)$ & \\
\hline Primary & $149(29.5 \mid)$ & $322(30.23)$ & $492(35.17)$ & I703(26.98) & \\
\hline Middle & $126(24.95)$ & $422(39.62)$ & $400(28.60)$ & 2839(44.98) & \\
\hline University & $37(7.34)$ & $162(15.22)$ & $99(7.07)$ & $1086(\mid 7.21)$ & \\
\hline Marital status (n, \%) & & & & & $<0.0001$ \\
\hline Marriage & $408(80.95)$ & $932(87.60)$ & II84(84.75) & $5729(90.83)$ & \\
\hline Divorced or widowed & $96(19.05)$ & $132(12.40)$ & $213(15.25)$ & $578(9.17)$ & \\
\hline Smoking status (n, \%) & & & & & 0.0007 \\
\hline Never smoking & $352(69.70)$ & $699(65.63)$ & $978(69.91)$ & $4075(64.54)$ & \\
\hline Current smoking & $42(8.31)$ & $107(10.05)$ & $148(\mid 0.57)$ & $745(11.80)$ & \\
\hline Smoking before & III(2I.99) & $259(24.32)$ & $273(19.52)$ & $1494(23.66)$ & \\
\hline Alcohol consumption ( $\mathrm{n}, \%)$ & & & & & $<0.0001$ \\
\hline Never & $422(83.56)$ & $823(77.28)$ & I I06(79.06) & $4720(74.75)$ & \\
\hline Current & $23(4.55)$ & $94(8.82)$ & $145(10.36)$ & $834(|3.2|)$ & \\
\hline Quit & $60(11.89)$ & $148(13.90)$ & $148(10.58)$ & $760(12.04)$ & \\
\hline \multicolumn{6}{|l|}{ Long-time bedridden ( $\mathrm{n}, \%)$} \\
\hline No & $446(88.32)$ & $968(90.89)$ & $1379(98.57)$ & 6255(99.06) & \\
\hline Yes & $59(11.68)$ & $97(9.11)$ & $20(1.43)$ & $59(0.94)$ & \\
\hline Vision (n, \%) & & & & & $<0.001$ \\
\hline Yes & $147(29.10)$ & $286(26.85)$ & $306(21.87)$ & $1172(18.56)$ & \\
\hline
\end{tabular}

(Continued) 
Table 2 (Continued).

\begin{tabular}{|c|c|c|c|c|c|}
\hline Variables & $\begin{array}{l}\text { Frailty and } \\
\text { Cognitive } \\
\text { Impairment } \\
(505)\end{array}$ & $\begin{array}{l}\text { Frailty Only } \\
(1065)\end{array}$ & $\begin{array}{l}\text { Cognitive } \\
\text { Impairment } \\
\text { Only (I399) }\end{array}$ & $\begin{array}{l}\text { Nonfrail and with } \\
\text { Normal Cognitive } \\
\text { Function (6314) }\end{array}$ & P-value \\
\hline No & $358(70.90)$ & $779(73.14)$ & $1093(78.13)$ & $5142(81.44)$ & \\
\hline Hearing (n, \%) & & & & & $<0.0001$ \\
\hline Yes & $129(25.54)$ & $250(23.47)$ & $259(|8.5|)$ & $1023(16.20)$ & \\
\hline No & $376(74.46)$ & $815(76.52)$ & $1140(81.49)$ & $5291(83.80)$ & \\
\hline Urinary function $(\mathrm{n}, \%)$ & & & & & $<0.0001$ \\
\hline Dysfunction & $110(21.78)$ & $222(20.85)$ & $166(11.87)$ & $790(|2.5|)$ & \\
\hline Normal & $395(78.22)$ & $843(79.15)$ & $1233(88.13)$ & $5524(87.49)$ & \\
\hline Depression (n, \%) & & & & & $<0.0001$ \\
\hline No & $289(58.03)$ & $634(60.21)$ & II 45(82.55) & $5639(90.14)$ & \\
\hline Yes & $209(41.97)$ & $419(39.79)$ & $242(17.45)$ & $617(9.86)$ & \\
\hline MNA-SF (n, \%) & & & & & $<0.0001$ \\
\hline $12-14$ & $70(13.86)$ & $283(26.57)$ & $598(42.74)$ & $4 \mid 30(65.4 \mid)$ & \\
\hline $8-11$ & $232(45.94)$ & $475(44.60)$ & $656(46.89)$ & $|86|(29.47)$ & \\
\hline $0-7$ & $203(40.20)$ & $307(28.83)$ & $145(10.36)$ & $323(5.12)$ & \\
\hline Low Hand grip strength (n, \%) & & & & & $<0.001$ \\
\hline Yes & $422(83.56)$ & $74 \mid(69.58)$ & $856(61.19)$ & $2723(43.13)$ & \\
\hline No & $83(16.44)$ & $324(30.42)$ & $543(38.81)$ & $3591(56.87)$ & \\
\hline $\begin{array}{l}\text { The length of hospital stay } \\
\text { (mean, SD) }\end{array}$ & I I. $.02 \pm 7.23$ & $11.33 \pm 7.99$ & $9.52 \pm 6.94$ & $9.31 \pm 7.11$ & $<0.0001$ \\
\hline
\end{tabular}

Abbreviations: MNA-SF, Mini-Nutritional Assessment-Short Form (normal nutritional status: 12-14; at risk of malnutrition: 8-II; malnourished:0-7); ADL, activities of daily living.

\section{Stratified Association Between Cognitive Frailty Status and 30-Day Mortality by Gender}

This association between cognitive frailty status and 30-day mortality was almost unchanged by gender (female versus male), with a significantly greater risk of 30-day mortality in participants with cognitive frailty in both females and males. Detailed information is shown in Figure 2.

\section{Prognostic Score Comparison}

Our study shows that the identification of 30-day mortality was significantly higher using cognitive frailty $(\mathrm{AUC}=0.676$
[95\% CI: $0.621-0.731])$ than either frailty $(\mathrm{AUC}=0.644[95 \%$ CI: $0.594-0.694]$ ) or cognitive impairment (AUC $=0.606[95 \%$ CI: 0.556-0.655]) alone. In addition, there was a significant difference when the AUC of cognitive frailty was compared to that of cognitive impairment $(P=0.001)$ or frailty $(P=0.025)$ alone. However, the difference in AUC between frailty alone and cognitive impairment alone was not significant (0.193). The results are shown in Figure 3.

\section{Discussion}

Our study found that Chinese inpatients with cognitive frailty or frailty had an increased risk of 30-day mortality 
Table 3 Univariate Analysis for the Variables Associated with 30Day Mortality

\begin{tabular}{|c|c|c|c|}
\hline Variables & $\begin{array}{l}\text { Survivor at } \\
\text { 30-Day }\end{array}$ & $\begin{array}{l}\text { Deceased } \\
\text { at 30-Day }\end{array}$ & P-value \\
\hline Age (years) (mean, SD) & $72.4 \pm 5.7$ & $73.8 \pm 6.1$ & 0.014 \\
\hline \multicolumn{4}{|l|}{ Gender (n, \%) } \\
\hline Female & $385 I(41.93)$ & $40(40.40)$ & 0.759 \\
\hline Male & $5333(58.07)$ & $59(59.60)$ & \\
\hline Education (n, \%) & & & $<0.0001$ \\
\hline Illiterate & $1423(15.50)$ & $2 I(2 I .2 I)$ & \\
\hline Primary & $2622(28.56)$ & $44(44.44)$ & \\
\hline Meddle & $3758(40.93)$ & $29(29.29)$ & \\
\hline University & $1379(15.02)$ & $5(5.05)$ & \\
\hline ADL (mean, SD) & $27.65 \pm 4.37$ & $28.04 \pm 4.12$ & $<0.0001$ \\
\hline BMI $\left(\mathrm{kg} / \mathrm{m}^{2}\right)$ (mean, SD) & $23.65 \pm 3.70$ & $23.57 \pm 3.34$ & 0.2596 \\
\hline $\begin{array}{l}\text { Frailty cognitive } \\
\text { category }(\mathrm{n}, \%)\end{array}$ & & & $<0.0001$ \\
\hline Cognitive frailty & $479(5.22)$ & $26(26.26)$ & \\
\hline Only Frailty & 1046(II.39) & $19(19.19)$ & \\
\hline $\begin{array}{l}\text { Only cognitive } \\
\text { impairment }\end{array}$ & $1384(\mid 5.07)$ & $15(15.15)$ & \\
\hline $\begin{array}{l}\text { No frailty and no } \\
\text { cognitive impairment }\end{array}$ & $6275(68.33)$ & $39(39.39)$ & \\
\hline Ethnicity (n, \%) & & & 0.256 \\
\hline Han & $8682(94.53)$ & $91(91.92)$ & \\
\hline Other & $502(5.47)$ & $8(8.08)$ & \\
\hline Marital status (n, \%) & & & 0.717 \\
\hline Marriage & $8166(89.02)$ & $87(87.88)$ & \\
\hline Divorced or widowed & $1007(10.98)$ & $12(12.12)$ & \\
\hline Smoking status (n, \%) & & & 0.204 \\
\hline Never smoking & 6046(99.05) & $58(58.59)$ & \\
\hline Current smoking & $1026(98.46)$ & $16(16.16)$ & \\
\hline Smoking before & $2112(98.83)$ & $25(25.25)$ & \\
\hline $\begin{array}{l}\text { Alcohol consumption ( } \mathrm{n} \text {, } \\
\% \text { ) }\end{array}$ & & & 0.444 \\
\hline Never & 6999(76.21) & $72(72.73)$ & \\
\hline Current & $1085(11.81)$ & $\mathrm{II}(\mathrm{II} . \mathrm{II})$ & \\
\hline Quit & $1100(11.98)$ & $16(16.16)$ & \\
\hline
\end{tabular}

(Continued)
Table 3 (Continued).

\begin{tabular}{|c|c|c|c|}
\hline Variables & $\begin{array}{l}\text { Survivor at } \\
\text { 30-Day }\end{array}$ & $\begin{array}{l}\text { Deceased } \\
\text { at 30-Day }\end{array}$ & P-value \\
\hline $\begin{array}{l}\text { Long-time bedridden } \\
(\mathrm{n}, \%)\end{array}$ & & & 0.003 \\
\hline No & $8957(97.65)$ & $91(91.92)$ & \\
\hline Yes & $227(2.47)$ & $8(8.08)$ & \\
\hline Vision (n, \%) & & & 0.06 \\
\hline Yes & $1898(20.67)$ & $13(13.13)$ & \\
\hline No & $7286(79.33)$ & $86(86.87)$ & \\
\hline Hearing (n, \%) & & & 0.939 \\
\hline Yes & $1643(17.89)$ & $18(18.18)$ & \\
\hline No & $754 I(82.1 I)$ & $81(81.82)$ & \\
\hline Urinary function $(\mathrm{n}, \%)$ & & & 0.340 \\
\hline Dysfunction & $|27|(\mid 3.84)$ & $17(17.17)$ & \\
\hline Normal & $7913(86.16)$ & $82(82.83)$ & \\
\hline Frailty $(\mathrm{n}, \%)$ & & & $<0.000$ I \\
\hline NO & $7659(83.40)$ & $54(54.55)$ & \\
\hline Yes & $1525(16.60)$ & $45(45.45)$ & \\
\hline $\begin{array}{l}\text { Cognitive impairment } \\
(\mathrm{n}, \%)\end{array}$ & & & $<0.0001$ \\
\hline No & $732 I(79.7 I)$ & $58(58.59)$ & \\
\hline Yes & $1863(20.29)$ & $4 I(4 I .4 I)$ & \\
\hline Depression (n, \%) & & & $<0.0001$ \\
\hline No & $764 I(84.00)$ & $66(67.35)$ & \\
\hline Yes & $1455(16.00)$ & $32(32.65)$ & \\
\hline MNA-SF (n, \%) & & & $<0.001$ \\
\hline $12-14$ & $506 \mid(55.1 I)$ & $20(20.20)$ & \\
\hline $8-11$ & $3181(34.64)$ & $43(43.43)$ & \\
\hline $0-7$ & $942(10.26)$ & $36(36.36)$ & \\
\hline $\begin{array}{l}\text { Low Hand grip strength } \\
(\mathrm{n}, \%)\end{array}$ & & & $<0.000$ I \\
\hline Yes & $466 \mid(50.75)$ & $81(81.82)$ & \\
\hline No & $4523(49.25)$ & $18(18.18)$ & \\
\hline $\begin{array}{l}\text { The length of hospital } \\
\text { stay (mean, SD) }\end{array}$ & $9.45 \pm 6.81$ & $9.82 \pm 7.51$ & 0.016 \\
\hline
\end{tabular}

Abbreviations: MNA-SF, Mini-Nutritional Assessment-Short Form (normal nutritional status: 12-14; at risk of malnutrition: 8-11; malnourished: 0-7). 
Table 4 Multiple Variable Regression Analysis for 30-Day Mortality According to Status of Cognitive Impairment and Frailty

\begin{tabular}{|l|l|l|l|l|}
\hline Exposure & $\begin{array}{l}\text { Non-Adjusted (OR,95\%, } \\
\text { CI) }\end{array}$ & $\begin{array}{l}\text { Adjusted I (OR,95\%, } \\
\text { CI) }\end{array}$ & $\begin{array}{l}\text { Adjusted II (OR,95\%, } \\
\text { CI,) }\end{array}$ & $\begin{array}{l}\text { Adjusted III (OR,95\%, } \\
\text { CI) }\end{array}$ \\
\hline Nonfrail and no cognitive & Reference & Reference & Reference & Reference \\
\hline Cognitive impairment only & $1.80(0.99-3.28)$ & $1.54(0.83-2.85)$ & $1.50(0.8 I-2.78)$ & $1.43(0.77-2.65)$ \\
\hline Frailty only & $2.88(I .63-5.06)$ & $2.7 I(I .53-4.78)$ & $2.39(I .32-4.34)$ & $1.85(I .01-3.4 I)$ \\
\hline $\begin{array}{l}\text { Frailty and cognitive } \\
\text { impairment }\end{array}$ & $6.83(3.9 I-1 I .95)$ & $5.73(3.16-10.36)$ & $5.02(2.7 I-9.33)$ & $3.43(I .80-6.55)$ \\
\hline
\end{tabular}

Notes: Adjusted I: age gender education. Adjusted II: age gender education, depression. Adjusted III: age gender education, depression, and ADL.

compared to those without frailty and cognitive impairment. However, the association in participants with only cognitive impairment compared to patients without frailty and cognitive impairment was not found to be significant, indicating that the combined effect of frailty and cognitive impairment was more hazardous for patients. Moreover, we found that cognitive frailty had better discrimination performance for 30-day mortality than frailty or cognitive impairment alone. Additionally, stratified analysis based on gender showed similar results. These findings highlight that early screening for frailty coexisting with cognitive impairment for older inpatients is essential. Given that these two geriatric syndromes undergo a dynamic change from prefrailty to frailty, it is crucial to perform effective interventions, such as prescribing exercise and nutritional programs for this vulnerable elderly population.

The prevalence of cognitive frailty was $5.44 \%$, which is slightly higher than that in a previous study conducted in a community setting. In a study reported by Shimada et al with 5104 community-dwelling older adults, the prevalence of cognitive frailty, measured by the Mini-Mental
State Examination (MMSE), and the frailty phenotype was $2.7 \%{ }^{29}$ In another study conducted with 8864 older adults, the prevalence of cognitive frailty was $1.2 \%{ }^{30}$ Furthermore, in a study that used modified CHS criteria and MMSE to examine frailty and cognitive impairment, the prevalence of cognitive impairment was $4.4 \%$, ${ }^{31}$ which was close to our study. However, another study combined prefrailty and frailty to define cognitive frailty, resulting in a higher prevalence of $22 \% .^{32}$ Similar results were found in a recent study that included prefrailty and frailty, with a prevalence of $22.6 \%,{ }^{8}$ which was higher than our results. Therefore, different subjects, definitions and measurement tools of frailty and cognitive impairment can lead to divergent prevalence results. Given the different definitions of cognitive frailty, further studies need to define a standard definition that will help to guild clinical staff to prevent and treat cognitive impairment.

A great number of studies have been published exploring this association, with a majority of these studies based on community-dwelling older adults ${ }^{13,15,33}$ or nursing home residents. $^{22}$ A study from Singapore Longitudinal

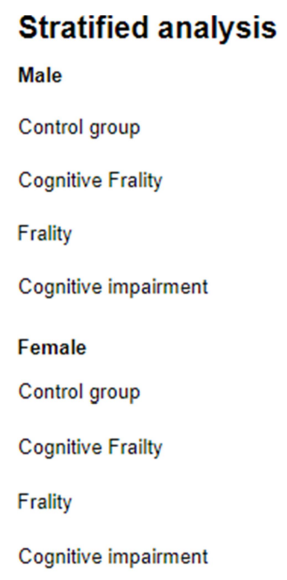

Odds Ration(OR, 95\% Cl)

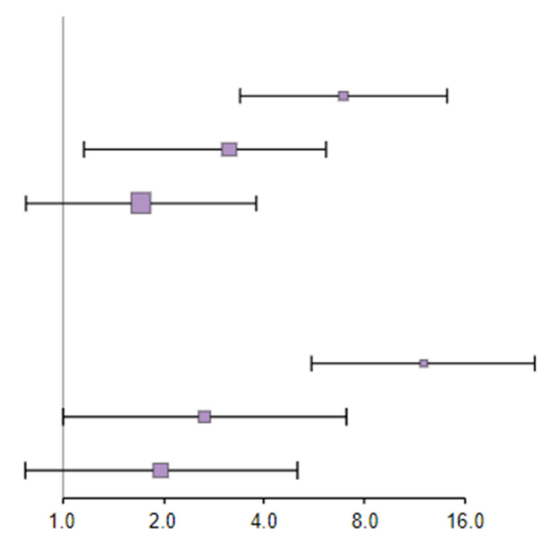

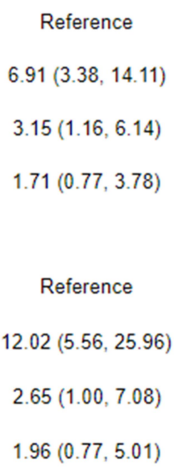

Reference

$3.15(1.16,6.14)$

$1.71(0.77,3.78)$

Reference

$2(5.56,25.96)$

$1.96(0.77,5.01)$

Figure 2 Stratified analysis between cognitive frailty status and 30-day mortality by gender. 


\section{ROC curve for 30-day mortality}

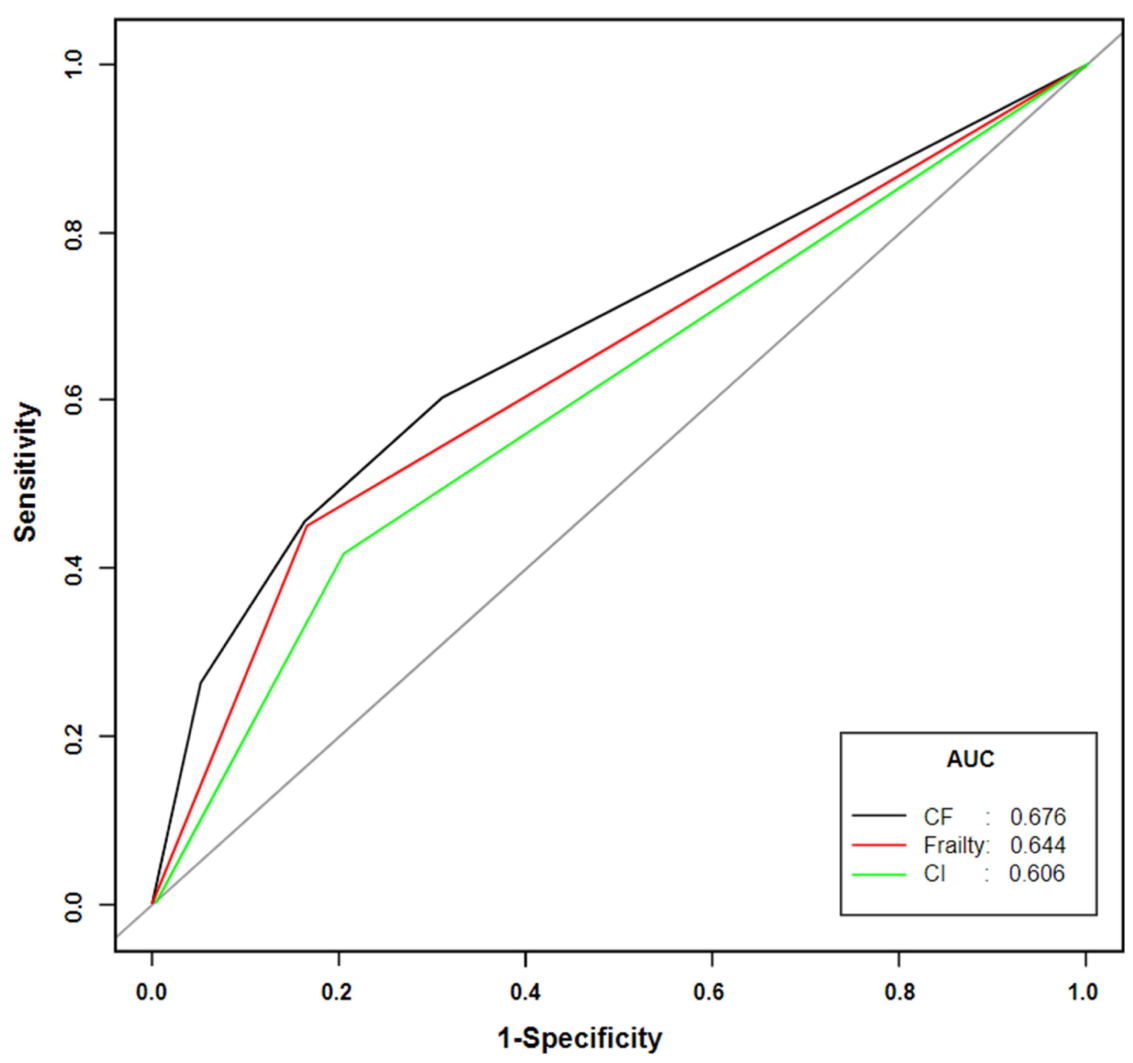

\section{CF: Cognitive frailty}

\section{Cl: Cognitive impairment}

Figure 3 Three prediction models for 30-day mortality based on receiver operation characteristic. Model I for cognitive frailty (black); Model 2 for frailty alone; Model 3 for cognitive impairment alone.

Aging (SLA), including 2375 community-dwelling older people, reported that the cognitive frailty group had an approximately 5 -fold greater risk of mortality when compared to the robust without cognitive impairment group. ${ }^{11}$ In addition, a cohort study with 3677 noninstitutionalized older people reported that the hazard ratio for mortality was 1.69 (95\% CI: 1.43-2.01) among cognitive frailty patients compared with robust individuals without cognitive impairment. ${ }^{8}$ Our findings indicated that patients with cognitive frailty had the strongest factor associated with 30-day mortality risk (3.43-fold) compared to those without frailty and cognitive impairment, which is consistent with these previously mentioned studies. However, our study focused on older adults in hospitals, and previously mentioned studies were community-dwelling older people.
In fact, older hospitalized patients often suffer from multiple comorbidities, leading to more vulnerability to the detrimental effects of cognitive frailty. Numerous older adults need to access the hospital to get to the root of their health problems. Cognitive frailty will become a serious issue that influences the health of hospitalized patients. A previous meta-analysis reported that older people with cognitive frailty had an increased risk of adverse outcomes, such as mortality, poor quality of life and hospitalization, than robust individuals without cognitive impairment. ${ }^{34}$ However, screening cognitive frailty is not routine clinical practice for hospitalized patients, resulting in neglect of the potential risk of cognitive frailty by medical staff. Therefore, our study and previous studies indicate the cumulative effects of combined frailty and 
cognitive impairment on adverse outcomes. It is very important that clinicians pay much more attention to these vulnerable patients.

A previous study explored the complicated pathways for cognitive frailty leading to death, indicating that inflammatory, vascular and hormonal factors may play an important role in coexisting frailty and cognitive impairment. ${ }^{35,36}$ Given the involvement of these complex mechanisms, more animal experiments and studies are needed to investigate the neurobiological pathways to determine the reason that cognitive frailty increases the risk of death. However, preventive and managerial measures for reversing cognitive impairment are a priority for clinicians and nurses. A previous study found that patients suffering from cognitive impairment who undertook physical activity were associated with a $36 \%$ reduction in mortality compared to physically inactive patients. $^{8}$ This study has important clinical implications. We speculate that regular physical activity improves muscle strength, which reduces mortality rates. Further evidence-based research is urgently required to understand this mechanism, guiding how to employ this effective intervention in a community- or hospital-based setting.

In our study, we need to be cautious when making recommendations to guide clinical practice. First, we collected data with a 30-day outcome, which is short-term. A longer follow-up is required to validate this association. Second, the design of the observational study was too limited to make a causal reference. Third, we used a simple cognitive tool that was suitable for application in a clinical setting, whereas numerous studies used MMSE ( $\leq 25$ points) to detect cognitive impairment; ${ }^{8,11}$ therefore, our results may generate a different prevalence of cognitive frailty that could not reflect multidimensional aspects of inpatients' capacity and function, leading to an underestimation of the risk of mortality. Fourth, other important confounding factors, such as the Charlson comorbidity index score, ${ }^{37}$ were not collected in the baseline survey, which may overestimate these results. Although this study has some limitations, several strengths also exist that merit attention. First, we adopted two-stage cluster sampling with a large number of participants from multiple centers, minimizing possible selection bias. Second, we used comprehensive statistical analysis, namely multivariate logistic regression analysis, to adjust for various covariates and we used stratified analysis to calculate all results.

\section{Conclusions and Implications}

In this study, we found that patients with cognitive frailty, a term describing a new concept of coexisting frailty and cognitive impairment, had an increased risk of 30-day mortality compared to older Chinese robust inpatients with intact cognitive function. This is a reminder that clinicians and nurses urgently need to screen for these two geriatric syndromes when older patients are admitted to the hospital. Furthermore, they need to plan for a comprehensive intervention, such as exercise and cognitive training, during hospitalization.

\section{Abbreviations}

ADL, activities of daily living; OR, odds ratio; MNA-SF, Mini-Nutritional Assessment-Short Form; CFS, Clinical Frailty Scale; MMSE, Mini-Mental State Examination; MoCA, Montreal Cognitive Assessment; CHS criteria, Cardiovascular Health Study; AUC, area under the curve; GDS, Geriatric Depression Scale GDS.

\section{Data Sharing Statement}

Deidentified data are accessible on reasonable request via e-mail to the corresponding author.

\section{Ethical Statement}

The study was approved by Ethics Committee of Peking Union Medical College Hospital (number S-K540). In addition, participants have provided written informed consent for this project. The authors confirm that this study was conducted in accordance with the Declaration of Helsinki.

\section{Author Contributions}

All authors contributed to data analysis, drafting or revising the article, have agreed on the journal to which the article will be submitted, gave final approval of the version to be published, and agree to be accountable for all aspects of the work.

\section{Funding}

Peking Union Medical College funded this study, with grant number 2018PT33001.

\section{Disclosure}

The authors declare no competing interests in this work.

\section{References}

1. Panza F, Lozupone M, Solfrizzi V, et al. Different cognitive frailty models and health- and cognitive-related outcomes in older age: from epidemiology to prevention. J Alzheimers Dis. 2018;62(3):993-1012. doi:10.3233/JAD-170963 
2. Clegg A, Young J, Iliffe S, Rikkert MO, Rockwood K. Frailty in elderly people. Lancet (London, England). 2013;381(9868):752-762. doi:10.1016/S0140-6736(12)62167-9

3. Ganguli M, Chang CC, Snitz BE, Saxton JA, Vanderbilt J, Lee CW. Prevalence of mild cognitive impairment by multiple classifications: the Monongahela-Youghiogheny Healthy Aging Team (MYHAT) project. Am J Geriatr Psychiatr. 2010;18(8):674-683. doi:10.1097/ JGP.0b013e3181cdee4f

4. Cunha AIL, Veronese N, de Melo Borges S, Ricci NA. Frailty as a predictor of adverse outcomes in hospitalized older adults: a systematic review and meta-analysis. Ageing Res Rev. 2019;56:100960. doi:10.1016/j.arr.2019.100960

5. Kallenberg MH, Kleinveld HA, Dekker FW, et al. Functional and cognitive impairment, frailty, and adverse health outcomes in older patients reaching ESRD-a systematic review. Clin J Am Soc Nephrol. 2016;11(9):1624-1639. doi:10.2215/CJN.13611215

6. Fougère $B$, Daumas $M$, Lilamand $M$, et al. Association between frailty and cognitive impairment: cross-sectional data from toulouse frailty day hospital. J Am Med Dir Assoc. 2017;18(11):990.e991-990. e995. doi:10.1016/j.jamda.2017.06.024

7. Robertson DA, Savva GM, Kenny RA. Frailty and cognitive impairment-a review of the evidence and causal mechanisms. Ageing Res Rev. 2013;12(4):840-851. doi:10.1016/j.arr.2013.06.004

8. Esteban-Cornejo I, Cabanas-Sánchez V, Higueras-Fresnillo S, et al. Cognitive frailty and mortality in a national cohort of older adults: the role of physical activity. Mayo Clin proc. 2019;94 (7):1180-1189.

9. Kelaiditi E, Cesari M, Canevelli M, et al. Cognitive frailty: rational and definition from an (I.A.N.A./I.A.G.G.) international consensus group. J Nutr Health Aging. 2013;17(9):726-734. doi:10.1007/ s12603-013-0367-2

10. Bieniek J, Wilczyński K, Szewieczek J. Fried frailty phenotype assessment components as applied to geriatric inpatients. Clin Interv Aging. 2016;11:453-459. doi:10.2147/CIA.S101369

11. Feng L, Zin Nyunt MS, Gao Q, Feng L, Yap KB, Ng TP. Cognitive frailty and adverse health outcomes: findings from the Singapore Longitudinal Ageing Studies (SLAS). J Am Med Dir Assoc. 2017;18(3):252-258. doi:10.1016/j.jamda.2016.09.015

12. Cano C, Samper-Ternent R, Al Snih S, Markides K, Ottenbacher KJ. Frailty and cognitive impairment as predictors of mortality in older Mexican Americans. J Nutr Health Aging. 2012;16(2):142-147. doi:10.1007/s12603-011-0104-7

13. St John PD, Tyas SL, Griffith LE, Menec V. The cumulative effect of frailty and cognition on mortality - results of a prospective cohort study. Int Psychogeriatr. 2017;29(4):535-543. doi:10.1017/ S1041610216002088

14. Aprahamian I, Suemoto CK, Aliberti MJR, et al. Frailty and cognitive status evaluation can better predict mortality in older adults? Arch Gerontol Geriatr. 2018;77:51-56. doi:10.1016/j. archger.2018.04.005

15. Lee WJ, Peng LN, Liang CK, Loh CH, Chen LK. Cognitive frailty predicting all-cause mortality among community-living older adults in Taiwan: a 4-year nationwide population-based cohort study. PLoS One. 2018;13(7):e0200447. doi:10.1371/journal. pone. 0200447

16. Hao Q, Dong B, Yang M, Dong B, Wei Y. Frailty and cognitive impairment in predicting mortality among oldest-old people. Front Aging Neurosci. 2018;10:295. doi:10.3389/fnagi.2018.00295

17. Liu LK, Chen CH, Lee WJ, et al. Cognitive frailty and its association with all-cause mortality among community-dwelling older adults in Taiwan: results from I-Lan longitudinal aging study. Rejuvenation Res. 2018;21(6):510-517. doi:10.1089/rej.2017.2038

18. Makhani SS, Kim FY, Liu Y, et al. Cognitive impairment and overall survival in frail surgical patients. $J$ Am Coll Surg. 2017;225(5):590600.e591. doi:10.1016/j.jamcollsurg.2017.07.1066
19. Downer B, Al Snih S, Howrey BT, Raji MA, Markides KS, Ottenbacher KJ. Combined effects of cognitive impairment and pre-frailty on future frailty and death in older Mexican Americans. Aging Ment Health. 2019;23(10):1405-1412. doi:10.1080/ 13607863.2018.1493719

20. Thein FS, Li Y, Nyunt MSZ, Gao Q, Wee SL, Ng TP. Physical frailty and cognitive impairment is associated with diabetes and adversely impact functional status and mortality. Postgrad Med. 2018;130 (6):561-567. doi:10.1080/00325481.2018.1491779

21. Solfrizzi V, Scafato E, Seripa D, et al. Reversible cognitive frailty, dementia, and all-cause mortality. the italian longitudinal study on aging. J Am Med Dir Assoc. 2017;18(1):89.e81-89.e88. doi:10.1016/ j.jamda.2016.10.012

22. Matusik P, Tomaszewski K, Chmielowska K, et al. Severe frailty and cognitive impairment are related to higher mortality in 12-month follow-up of nursing home residents. Arch Gerontol Geriatr. 2012;55(1):22-24. doi:10.1016/j.archger.2011.06.034

23. Jiao J, Wang Y, Zhu C, et al. Prevalence and associated factors for frailty among elder patients in China: a multicentre cross-sectional study. BMC Geriatr. 2020;20(1):100. doi:10.1186/s12877-020-14961

24. Aprahamian I, Cezar NOC, Izbicki R, et al. Screening for frailty with the FRAIL scale: a comparison with the phenotype criteria. $J \mathrm{Am}$ Med Dir Assoc. 2017;18(7):592-596. doi:10.1016/j. jamda.2017.01.009

25. Nasreddine ZS, Phillips NA, Bédirian V, et al. The montreal cognitive assessment, MoCA: a brief screening tool for mild cognitive impairment. J Am Geriatr Soc. 2005;53(4):695-699. doi:10.1111/ j.1532-5415.2005.53221.x

26. Marc LG, Raue PJ, Bruce ML. Screening performance of the 15 -item geriatric depression scale in a diverse elderly home care population. Am J Geriatr Psychiatr. 2008;16(11):914-921. doi:10.1097/ JGP.0b013e318186bd67

27. Soysal P, Veronese N, Arik F, Kalan U, Smith L, Isik AT. Mini nutritional assessment scale-short form can be useful for frailty screening in older adults. Clin Interv Aging. 2019;14:693-699. doi:10.2147/CIA.S196770

28. Pascual JC, Belinchón I, Ramos JM. Use of the Barthel index, activities of daily living, in dermatologic surgery in patients aged 80 years and older. Int $J$ Dermatol. 2015;54(2):222-226. doi:10.1111/ijd.12680

29. Shimada H, Makizako H, Doi T, et al. Combined prevalence of frailty and mild cognitive impairment in a population of elderly Japanese people. J Am Med Dir Assoc. 2013;14(7):518-524. doi:10.1016/j. jamda.2013.03.010

30. Shimada H, Makizako H, Lee S, et al. Impact of cognitive frailty on daily activities in older persons. $J$ Nutr Health Aging. 2016;20 (7):729-735. doi:10.1007/s12603-016-0685-2

31. Roppolo M, Mulasso A, Rabaglietti E. Cognitive frailty in italian community-dwelling older adults: prevalence rate and its association with disability. $J$ Nutr Health Aging. 2017;21(6):631-636. doi:10.1007/s12603-016-0828-5

32. Delrieu J, Andrieu S, Pahor M, et al. Neuropsychological profile of "cognitive frailty" subjects in MAPT study. J Prev Alzheimers Dis. 2016;3(3):151-159. doi:10.14283/jpad.2016.94

33. Lee Y, Kim J, Chon D, et al. The effects of frailty and cognitive impairment on 3-year mortality in older adults. Maturitas. 2018;107:50-55. doi:10.1016/j.maturitas.2017.10.006

34. Bu Z, Huang A, Xue M, Li Q, Bai Y, Xu G. Cognitive frailty as a predictor of adverse outcomes among older adults: a systematic review and meta-analysis. Brain Behav. 2020;e01926.

35. Halil M, Cemal Kizilarslanoglu M, Emin Kuyumcu M, Yesil Y, Cruz Jentoft AJ. Cognitive aspects of frailty: mechanisms behind the link between frailty and cognitive impairment. $J$ Nutr Health Aging. 2015;19(3):276-283. doi:10.1007/s12603-014-0535-z 
36. Vella Azzopardi R, Beyer I, Vermeiren S, et al. Increasing use of cognitive measures in the operational definition of frailty-A systematic review. Ageing Res Rev. 2018;43:10-16. doi:10.1016/j.arr.2018.01.003
37. Saraiva MD, Rangel LF, Cunha JLL, et al. Prospective GERiatric Observational (ProGERO) study: cohort design and preliminary results. BMC Geriatr. 2020;20(1):427. doi:10.1186/s12877-020-01820-4

\section{Publish your work in this journal}

Clinical Interventions in Aging is an international, peer-reviewed journal focusing on evidence-based reports on the value or lack thereof of treatments intended to prevent or delay the onset of maladaptive correlates of aging in human beings. This journal is indexed on PubMed Central, MedLine, CAS, Scopus and the Elsevie
Bibliographic databases. The manuscript management system is completely online and includes a very quick and fair peer-review system, which is all easy to use. Visit http://www.dovepress.com/ testimonials.php to read real quotes from published authors.

Submit your manuscript here: https://www.dovepress.com/clinical-interventions-in-aging-journal 